\title{
Adaptive Moblile Spot Diffusing Transmiter for an Indoor Optical Wireless System
}

\author{
Jamal M. Alattar ${ }^{+}$and Jaafar M. H. Elmirghani ${ }^{\dagger}$ \\ Institute of Advanced Telecommunications \\ Swansea University \\ Singleton Park, Swansea SA2 8PP, UK \\ +e-mail: 181853@swan.ac.uk \\ †e-mail:J.M.H.Elmirghani@swan.ac.uk
}

\begin{abstract}
An adaptive approach is used to adjust the transmit power of each beam in a line strip multi-beam optical transmitter based on information about the quality of the received signal due to each spot. The system assumes a feedback link between the optical transceivers. Diversity detection is employed to combat the degrading effect of the background noise in the channel with a 7detectors angle diversity receiver. Both the optical transmitter and the multibranch receiver are fully mobile. The performance of the system is evaluated for two weakest links and compared against that of a non-adaptive spot diffusing transmitter. Our results show that the adaptive transmitter produces a considerable gain of $12.9 \mathrm{~dB}$ in signal-to-noise-ratio (SNR) when the distance separating the transceivers is largest in the case of a non mobile transmitter. Furthermore, a $6.8 \mathrm{~dB}$ increase in SNR is obtained for the mobile transmitter compared to the non-adaptive transmitter's scenario.
\end{abstract}

Keywords: Spot diffusing, mobile transmitter, multi-beam, optical wireless, indoor channel, adaptive.

\section{Introduction}

Optical wireless connectivity has recently emerged as a good candidate for indoor local area networks (LANs) and connection of computer peripherals, due to lack of interference as well as governmental regulation and inexpensive components. Optical wireless application growth is extending to outdoor laser links between buildings and campuses using portable terminals for high-data rate (hundreds of Mbps) links for internet backbones, transfer of medical, banking, and computer data, and other applications where high-data rate links are needed. Indoor links are still in need to upgrade and offer wireless communication with high quality signals and meet the increased data rates when portable devices are used.

Non directed line-of-sight (LOS) links, also known as "diffuse links" allow the system to operate even when barriers are placed between the transmitter and receiver; and are therefore becoming increasingly popular. A diffuse transmitter points vertically upwards towards the ceiling, emitting a wide beam of infrared energy and the receiver has a wide field-of-view (FOV), to enable it collect the signal from all reflective surfaces after it has undergone multiple reflections from the ceiling, walls and room objects. The propagation of the transmitted signals follows multiple paths before reaching the receiver's collection area in the indoor environment thus causing temporal 
dispersion on the received pulses. The pulse spread in turn causes the binary transmitted symbols to overlap which introduces Inter Symbol Interference (ISI) that, if not completely corrected, will result in erroneous message detection. Directive noise sources (natural and artificial) further impair the signal and reduce SNR.

A proven technique to mitigate these effects is to replace the fully diffuse optical transmitter by one that produces multiple narrow-beams casting small diffusing spots on the ceiling [1]-[3]. The narrow beams can be practically produced using a holographic optical diffuser mounted on the face of the transmitter [4], [5]. Holograms produced using computer methods can flexibly take advantage of varying the intensity of a particular spot and/or the intensity distribution of the spots. The spots, cast on the ceiling, become secondary Lambertian transmitters (diffusers). Further improvement in the signal quality in the indoor environment has been shown with the employment of angle diversity reception (with multiple photo detectors) to replace the single-detector wide field-of-view receiver traditionally used in conventional systems. This approach was mainly implemented to confine the signal rays to within the field-of-view of the receiver detectors as well as limit the amount of received background noise through the selection of an optimum reception direction. Such designs have detectors pointed to different directions and therefore can reduce the effects of ambient light noise. Various designs of angle diversity receivers have been considered including the three branch square-based fly-eye angle diversity receiver [6], [7], the hexagonal based 7-detector diversity receiver [7] - [9] and the multi-layered narrow FOV detectors composite angle diversity receiver [10]. Since noise is directional in this environment, circuitry in the optical receiver can implement signal combining techniques which enable the diversity receiver to combine its branch detectors' signals or select the one with best SNR. An efficient angle diversity receiver design has to produce a high and uniform SNR distribution within the room. Increasing the number of branches of the diversity receiver with an optical photo-detector on each has been shown to improve the gain achieved with spot diffusing configurations [7]. Combining the detected signals from each photo detector using maximum ratio combining (MRC) yields better output than either selecting the detector with the highest signal-to-noise-ratio (SNR) (selection combining) or equally combining all detectors' signals (equal gain combining, EGC).

In this work, we present a study of a novel approach to optimise signal reception with mobile transceivers. The method focuses on varying the power allocated to each beam in a single line strip multi-beam transmitter so as to optimise the received SNR for a given receiver location. A new adaptive method is proposed and used to set the amount of power transmitted on each beam based on information about the signal quality fed back to the transmitter by the receiver. Effectively, the proposed method varies the optical power in each beam or spot (secondary transmitter) in a manner that optimises the SNR at the receiver. As a result of the transmitter's mobility, the diffusing spots will change their locations [11],[12]. Spots that are closer to the receiver are to be given higher transmit powers than those far away from the receiver especially when the distance separating the transceivers becomes large (e.g., transmitter near one room corner and the receiver near an opposite room corner). The following proposed protocol is used to obtain the power distribution ratios among the line strip beams: periodically the transmitter switches on one of the beams in turn and the receiver measures the SNR due to that beam (diffusing spot). The process is 
repeated with the transmitter switching on a beam at a time. The receiver then transmits back to the transmitter at a low data rate (for reliability) the measured SNR. The transmitter then sets its beams power tap weights in proportion to the SNR. The tap weights are adjusted periodically depending on the degree of mobility anticipated in the environment. Our approach has achieved an SNR gain of as much as $12.9 \mathrm{~dB}$ without transmitter mobility and as high as $9.7 \mathrm{~dB}$ with transmitter mobility. Furthermore, an improvement of $6.8 \mathrm{~dB}$ is obtained at the weakest links.

\section{System Setup}

The optical wireless link is established on a communication plane (CP) $1 \mathrm{~m}$ above the floor in an empty mid-sized room (size: $4 \mathrm{~m} \times 8 \mathrm{~m} \times 3 \mathrm{~m}$ Width, Length, Height). The room has no doors or windows and the walls and ceiling plastering results in $80 \%$ reflectivity of incident light whereas the floor produces reflectivity of $30 \%$. A $1 \mathrm{~W}$ upright optical transmitter (elevation $=90^{\circ}$ ) is placed in the centre of the communication plane (at $\mathrm{x}=2 \mathrm{~m}$ and $\mathrm{y}=4 \mathrm{~m}$ ). Multiple narrow beams are produced with a holographic device mounted on the face of the optical transmitter resulting in a cluster of 80 equally-separated narrow beams casting a spot on the ceiling as depicted in Fig. 1. The power associated with each spot can be adjusted using a liquid crystal device for example.

The 7-detectors angle diversity receiver used has a hexagonal base as shown in Fig. 2. One detector is placed on its top and one on each of the six elevated branches with $20^{\circ}$ elevation angle. The side detectors have symmetric azimuth angles with the $\mathrm{x}$ and $y$ axes. Hence the azimuth angles: $45^{\circ}, 90^{\circ}, 135^{\circ}, 225^{\circ}, 270^{\circ}$ and $315^{\circ}$ and $0^{\circ}$ respectively were used. The detectors' FOVs were optimised to achieve highest SNR from the line of diffusing spots. The detectors that face two walls (detectors 1, 3, 4 and 6) were given larger FOVs than those facing only one wall (detectors 2 and 5) so as to collect most of the signals reflected off both walls. Hence, the FOVs of the six detectors on the side branches were $60^{\circ}, 50^{\circ}, 60^{\circ}, 60^{\circ}, 50^{\circ}$ and $60^{\circ}$. The FOV of the top detector was chosen smaller $\left(24^{\circ}\right)$ in order to avoid the noise direct power component as the noise sources are directive.

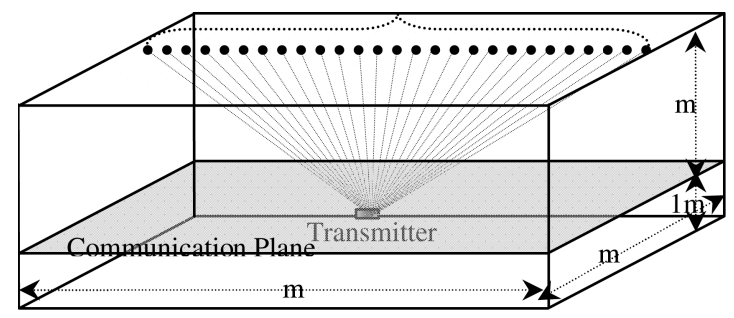

Fig. 1: Beam cluster spot diffusing transmitter model

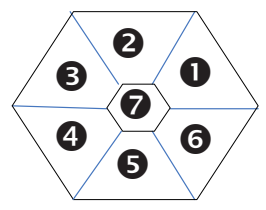

Fig. 2: 7-detectors angle diversity receiver (top view)

The background noise was evaluated using eight incandescent light sources placed equidistantly on the ceiling, $2 \mathrm{~m}$ apart (along the lines $\mathrm{x}=1 \mathrm{~m}$ and $\mathrm{x}=3 \mathrm{~m}$ ) starting at $\mathrm{x}$ $=1 \mathrm{~m}$ and $\mathrm{y}=1 \mathrm{~m}$; thus producing a well-illuminated environment. Each lamp is of type 
Philips PAR 38 which emits an optical power of $65 \mathrm{~W}$ where the mode number $\mathrm{n}=$ 33.1.

Ray-tracing simulation was used to compute the received optical signal as well as the background noise following the algorithm introduced by Barry et al. [13]; the simulation was developed in $\mathrm{C}++$. Simulation was carried out along two lines on the communication plane: one closer to a wall, $x=1 \mathrm{~m}$ (where the signal reception is expected to be influenced by reflections) and the other along the middle line of the CP, $\mathrm{x}=2 \mathrm{~m}$, with $1 \mathrm{~m}$ receiver position intervals. Results were produced for 14 different receiver locations (starting $1 \mathrm{~m}$ away from the wall). Due to symmetry in the room, results for the line $\mathrm{x}=3 \mathrm{~m}$ will be the same as for the line $\mathrm{x}=1 \mathrm{~m}$.

\section{System Analysis}

\subsection{Mobile transmitter analysis}

The evenly spaced beams are produced with equal emitted angles in one direction. Based on the fact that as the transmitter moves around on the communication plane, the beams' angles remain unchanged, the new coordinates of the spots can be computed with respect to the original transmitter location (at the centre of the communication plane). As the transmitter approaches a wall, some spots will start appearing on the wall as their beams get intercepted by that wall, Fig. 3. A transmitter's motion in either the $\mathrm{y}$ or $\mathrm{x}$ direction causes spots to fall on the wall that the transmitter is approaching. When the transmitter moves in the y direction, for example, (across room length) the diffusing spots will move away from one wall and become closer to the other and vice versa. Further transmitter movement will cause some spots to move from the ceiling and appear on the wall.

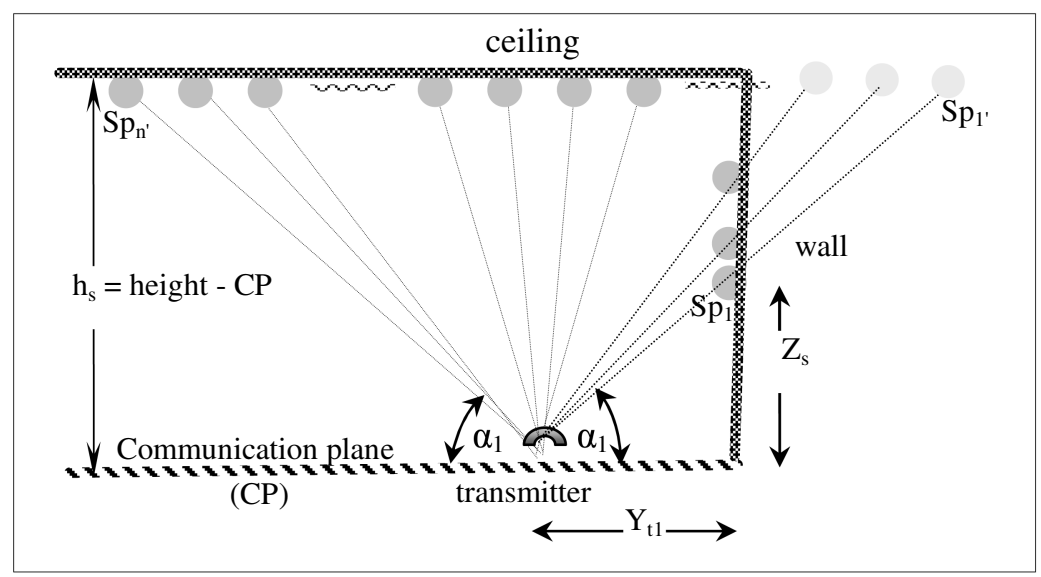

Fig. 3: Mobile transmitter scenario 
Therefore, as shown in Fig. 3, the new vertical distance $\mathrm{Z}_{\mathrm{s}}$ (from the CP) of the spot that appears on a wall is found from

$$
\mathrm{Z}_{\mathrm{s}}=\mathrm{Y}_{\mathrm{t} 1} \cdot \tan \left(\alpha_{\mathrm{i}}\right)
$$

where $Y_{t 1}$ is the new distance of the transmitter - on the y axis - from the wall it is moving to, and $\alpha_{\mathrm{i}}$ is the angle of beam $i$ with respect to the communication plane given by

$$
\tan \left(\alpha_{\mathrm{i}}\right)=\mathrm{h}_{\mathrm{s}} / \mathrm{d}_{\mathrm{yi}}
$$

where $\mathrm{h}_{\mathrm{s}}=$ height $-\mathrm{CP}=2 \mathrm{~m}$ and the distance $d_{y i}$ is found from

$$
d_{y i}=\frac{\text { length }}{2}-\left(\frac{\text { length }}{2 N_{y}}+\left(\frac{\text { length }}{N_{y}}\right) \cdot i\right), \quad 1 \leq i \leq \frac{N_{y}}{2}
$$

where length is the room length (dimensions along the y-axis) and the first spot is assumed to be length/ $2 N_{y}$ from the wall, and for the other half of the spots to the right of the transmitter

$$
d_{y i}=\left(\left(\frac{\text { length }}{N_{y}}\right) \cdot i-\frac{\text { length }}{2 N_{y}}\right)-\frac{\text { length }}{2}, \quad \frac{N_{y}}{2}+1 \leq i \leq N_{y}
$$

\subsection{Signal-to- noise ratio calculation}

The simplest modulation technique for OW systems is On-Off keying (OOK) which employs a rectangular pulse with duration equal to the bit period for each binary bit. The bit rate for our OW system is 50 Mbits/s giving a bit period of 20 ns. The SNR associated with the received signal is given by

$$
S N R=\left(\frac{R \times\left(P_{s 1}-P_{s 0}\right)}{\sigma_{t}}\right)^{2}
$$

where $R$ is the detector responsivity ( $R=0.5 \mathrm{~A} / \mathrm{W}$ in this study), $P_{s l}$ and $P_{s 0}$ are the powers associated with a logic " 1 " and logic " 0 " received signals respectively and $\sigma_{t}^{2}$ is the total noise variance which can be classified into three categories as

$$
\sigma_{t}^{2}=\sigma_{b n}^{2}+\sigma_{p r}^{2}+\sigma_{s}^{2}
$$

The first, $\sigma_{b n}$, is the shot noise induced by the background light which can be computed from its respective associated background noise power level $P_{b n}$ using

$$
\sigma_{\mathrm{bn}}=\sqrt{2 \times q \times P_{b n} \times R \times B W}
$$

where $q$ is the electron charge and BW is the receiver bandwidth.

The second noise component is the receiver noise generated in the preamplifier components. The preamplifier used in this work is the positive-intrinsic-negative bipolar-junction-transistor (PIN-BJT) design proposed by Elmirghani et al [14]. This 
preamplifier structure has a noise current density of $\sigma_{p r}=2.7 \mathrm{pA} / \sqrt{ } \mathrm{Hz}$ and a bandwidth of $70 \mathrm{MHz}$. Therefore, the preamplifier shot noise $\sigma_{p r}$ is

$$
\sigma_{\mathrm{pr}}=2.7 \times 10^{-12} \sqrt{70 \times 10^{6}}=0.023 \mu \mathrm{A}
$$

Finally, the noise induced by the received signal power $\sigma_{s}$, consists of two parts depending on the logical level of the received signal. The shot noise current is $\sigma_{s l}$ when a signal logic " 1 " is received and a different shot noise current $\sigma_{s} 0$ when a signal logic " 0 " is received. This signal dependant noise is very small in this work and therefore can be neglected.

Hence, the SNR can be calculated using equations (5) through (8) as

$$
S N R=\frac{\left(P_{s 1}-P_{s 0}\right)^{2}}{8 \times\left(\left(0.023 \times 10^{-6}\right)^{2}+70 \times 10^{6} \times q \times P_{b n}\right)}
$$

\subsection{Maximum ratio combining}

In an optical receiver with multiple branches, the signal collected from each branch detector is processed separately to produce the resulting output electrical signal. Circuitry integrated within the optical receiver has the purpose of either the selection of one branch detector or the combination (with some predefined criteria) of the detected optical signal from some or all branches. The select best scheme also known as selection combining (SC) chooses the branch with best SNR value. Two widely known combining techniques in diversity reception are the equal gain combining (EGC) and the maximum ratio combining (MRC).

While the EGC method adds the detected signals from all branches together, the MRC combines these signals according to weights proportional to their collected noises. It turns out that the EGC technique is a special case of the MRC with the combining weights set to unity (i.e., 1). For the MRC receiver, a signal multiplier circuit is added before the combiner circuit which takes its weight factor from the SNR estimator of a branch detector to produce the proportional gain of that branch. The maximal-ratio combiner circuit requires a variable gain amplifier per sector and a summing circuit. Clearly the differences between the combining methods are most noticeable as the unbalance in the distribution of the SNR among the sectors increases. Under the assumption of independent noise, the optimum output SNR is achieved by the maximal-ratio combining receiver [15]. The SNR using the Maximum Ratio Combining method is given by

$$
\mathrm{SNR}_{\text {MRC }}=\frac{\left(\sum_{i=1}^{J}\left(w_{i} \cdot I_{i}\right)\right)^{2}}{\sum_{i=1}^{J}\left(w_{i} \cdot \sigma_{i}\right)^{2}}, \quad 1=\mathrm{i}=\mathrm{J}
$$

where $\sigma_{\mathrm{i}}$ is the standard deviation of the total noise in branch $i$. 
Setting the weight $w$ to $\frac{I}{\sigma^{2}}$, it can be shown that the output SNR is

$$
\mathrm{SNR}_{\mathrm{MRC}}=\sum_{i=1}^{J}\left(\frac{I_{i}}{\sigma_{i}}\right)^{2}, \quad 1=\mathrm{i}=\mathrm{J}
$$

\section{Results}

Figure 4 shows the SNR distribution along two lines: the room centre line $x=2 \mathrm{~m}$ and its edge line, $x=1 \mathrm{~m}$. Results are given at two positions in addition to the reference position at the centre of the communication plane (at $2 \mathrm{~m}, 4 \mathrm{~m}, 1 \mathrm{~m}$ ). The results are quoted for an adaptive transmitter versus those obtained with a non-adaptive transmitter. The positions selected are at the two weakest links: near the corner at $(1 \mathrm{~m}$, $1 \mathrm{~m}, 1 \mathrm{~m})$ and near a side wall at $(2 \mathrm{~m}, 7 \mathrm{~m}, 1 \mathrm{~m})$. It is seen that the use of an adaptive transmitter produced enhancement in the performance of the spot diffusing $\mathrm{OW}$ system. This increase in SNR with an adaptive transmitter is seen to be more along the centre line than along the edge line. On the edge line, the line of spots lies directly underneath the noise sources whose influence on the system's performance is clear with lower SNR levels than along the room centre line. In spite of the strong influence and the directive nature of the noise sources, the employment of a line strip spot diffusing transmitter combined with a multi-detector angle diversity receiver produced a uniform SNR distribution across the length of the room.

The introduction of transmitter mobility alters this uniform SNR distribution. This can be seen in the plots where the highest SNR levels are where the distance between the transceivers is shortest and vice versa. The SNR levels with the adaptive transmitter at the farthest receiver positions from the transmitter have increased by as much as $6.8 \mathrm{~dB}$ as can be clearly seen from second plot of Fig. 4b. Although with the adaptive transmitter a considerable gain in SNR has been obtained even with transmitter mobility, some receiver positions did not benefit from this technique. When the transmitter is near the room corner; at $(1 \mathrm{~m}, 1 \mathrm{~m}, 1 \mathrm{~m})$ and the receiver at $\mathrm{y}=5 \mathrm{~m}(\mathrm{x}=$ $1 \mathrm{~m}$ and $\mathrm{x}=2 \mathrm{~m}$ ) for example, no gain in SNR is observed when the adaptive method is used. This is attributed to the small optical power collected from the diffusing spots as a result of the transmitter moving to the corner and the weak signal reflected off the wall as the receiver is now farthest from the wall (distance $=3 \mathrm{~m}$ ). The same can be said to explain the equal SNR levels for the case of transmitter at $(2 \mathrm{~m}, 7 \mathrm{~m}, 1 \mathrm{~m})$ and receiver at $\mathrm{x}=1 \mathrm{~m}$ and $\mathrm{y}=3 \mathrm{~m}$ when both adaptive and non adaptive transmitters are used. The effect of noise along the room centre line is minimal which produced higher SNR than along the room edge line. 

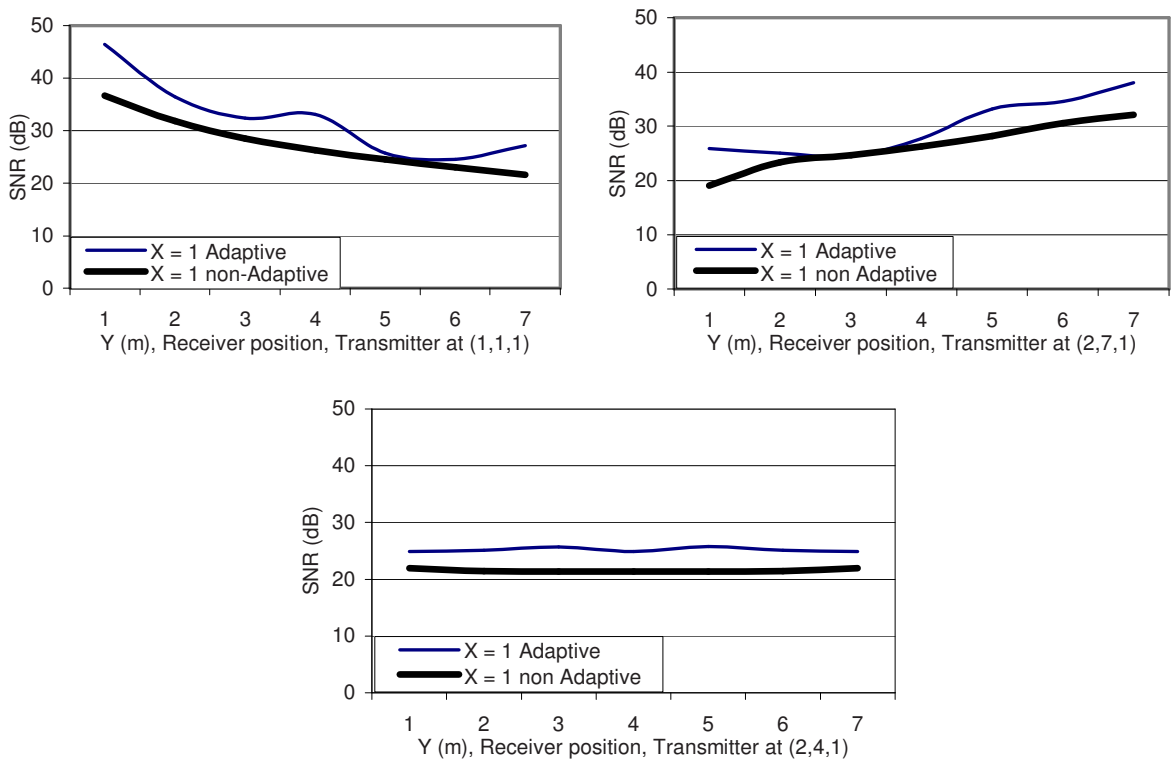

a) $\mathrm{x}=1 \mathrm{~m}$ line
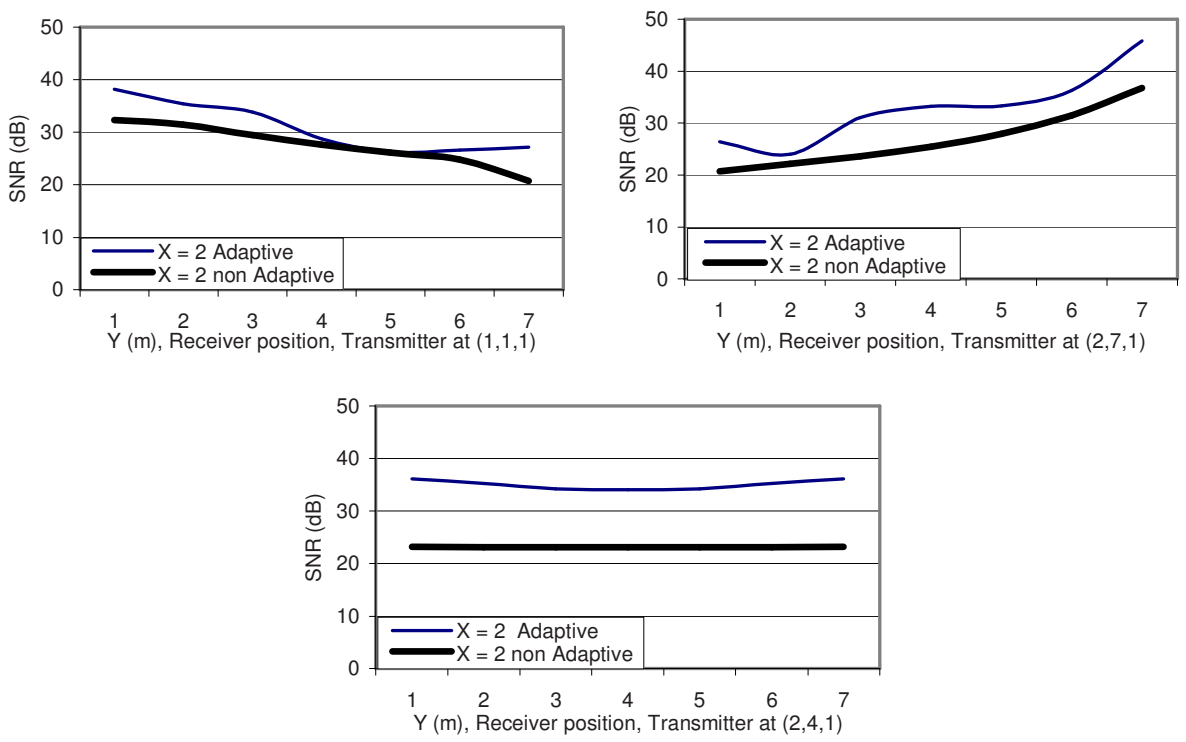

b) $x=2 m$ line

Fig. 4: Signal-to-noise ratio (SNR) comparisons of mobile spot diffusing transmitter across room length (adaptive and a non-adaptive)

Multipath reflection is seen to improve the received signal quality when comparing the SNR levels as the receiver is closer to a wall (at $\mathrm{y}=1 \mathrm{~m}$ and $7 \mathrm{~m}$ ) with that when it 
is away from the same wall (at $y=2 \mathrm{~m}$ and $6 \mathrm{~m}$ ). The plots of the SNR distributions due to the adaptive transmitter show a rise in SNR level when the receiver is near a wall and then a sloped decrease as it moves away towards the room centre where the multipath effect becomes less as reflected signals travel longer paths and the transmitter-receiver separation increases.

\section{Conclusions}

In this paper, we proposed an adaptive multi-spot diffusing transmitter and showed the advantage of employing the technique with a single line strip along with angle diversity reception. The aim was to adjust the transmit powers of the multi-beam transmitter relative to the receiver's location. Performance evaluation was reported for a system employing a 7-detectors angle diversity receiver. Furthermore, the adaptive transmitter approach was applied to a mobile spot diffusing transmitter and the results show a considerable performance improvement compared to the system employing a non adaptive transmitter. An SNR gain of $12.9 \mathrm{~dB}$ was obtained over the non adaptive transmitter without mobility and an SNR gain of over $6.8 \mathrm{~dB}$ was obtained at the weakest links when the non adaptive transmitter is mobile.

The use of angle diversity reception is very effective in reducing the effect of background noise. Employing a 7-detectors angle diversity with maximum ratio combining produced the highest uniform SNR distribution across the room length. The mobility of the spot diffusing transmitter influenced this distribution as the transceivers have maximum separation distance. Moreover, with the adaptive transmitter, receiver locations far away from both the spot diffusing transmitter and the room side wall showed no performance improvement compared to the non adaptive transmitter figures.

The multipath reflection increased the SNR gain when the adaptive transmitter was used. The delay spread and the optical path loss distribution of both systems were also investigated but not included in this study for brevity.

\section{References}

1. Pouyan Djahani and Joseph M. Kahn, "Analysis of Infrared Wireless Links Employing Multibeam Transmitters and Imaging Diversity Receivers," IEEE Trans. Commun., vol. 48, no. 12, pp. 2077-2088, Dec. 2000.

2. S. T. Jivokova and M. Kavehard: "Multispot diffusing configuration for wireless infrared access," IEEE Trans. Commun., vol. 48, pp. 970-978, Jun. 2000.

3. G. Yun and M. Kavehrad, "Spot diffusing and fly-eye receivers for indoor infrared wireless communications," in Proc. 1992 IEEE Conf. Selected Topics in Wireless Communications, Vancouver, BC, Canada, pp. 286-292, Jun. 1992.

4. M. R. Pakravan, E. Simova, and M. Kavehard, "Holographic diffusers for indoor infrared communication systems," Int. J. Wireless Inform. Networks, vol. 4, no. 4, pp. 259-274, 1997. 
5. P. L. Eardley, D. R. Wiely, D. Wood, and P. McKee, "Holograms for optical wireless LANs," IEE Proc. Optoelectron., vol. 143, no. 6, pp. 365-369, Dec. 1996.

6. A. G. Al-Ghamdi and J. M. H., Elmirghani, "Line Strip-Diffusing Transmitter Configuration for Optical Wireless Systems Influenced by Background Noise and Multipath Dispersion," IEEE Trans. Commun., vol. 52, no. 1, pp. 37-45, Jan. 2004.

7. A. G. Al-Ghamdi and J. M. H., Elmirghani, "Performance Evaluation of a Triangular Pyramidal Fly-Eye Diversity Detection for Optical Wireless Communications," IEEE Commun. Mag., pp. 80-86, Mar. 2003.

8. A. G. Al-Ghamdi and J. M. H., Elmirghani, "Performance evaluation of a pyramidal fly-eye diversity antenna in an indoor optical wireless multipath propagation environment under very directive noise sources," IEE Proc.Opto., vol. 150, no. 5, pp. 482-489, Oct. 2003.

9. A. G. Al-Ghamdi and J. M. H., Elmirghani, "Spot diffusing technique and angle diversity performance for high speed indoor diffuse infra-red wireless transmission," IEE Proc.-Optoelectron., Vol. 151, No. 1, pp. 46-52, Feb. 2004.

10. J. M. Alattar, J. M.H. Elmirghani, "Multi-line Multi-Spot Diffusing Indoor OW Channel with A 7-Detectors Diversity Receiver," Proc. of the London Communications Symposium, University College London, pp. 37-40, Sept. 2006.

11. A. G. Al-Ghamdi and J. M. H., Elmirghani, "Characterization of mobile spot diffusing optical wireless system with diversity receiver," IEEE Commun. Society, pp. 133-138, Jan. 2004.

12. J. M. Alattar, J. M.H. Elmirghani, "Evaluation of An Indoor OW Channel Employing A Mobile Multi-line Multi-Spot Diffusing Transmitter and A Seven Detectors Angle Diversity Receiver," Proc. of the London Communications Symposium, University College London, pp. 29-32, Sept. 2006.

13. John R. Barry, et al. "Simulation of Multipath Impulse Response for Indoor Wireless Optical Channels," IEEE Journal on selected areas in communication, Vol. 11, no. 3, pp. 367-379, Apr. 1993.

14. J. M. H. Elmirghani, H. H. Chan, and R. A. Cryan, "Sensitivity evaluation of optical wireless PPM systems utilising PIN-BJT receivers," IEE Proc.Optoelectron., vol. 143, no. 6, pp. 355-359, Dec. 1996.

15. Rui Tomaz Valadas and A. M. de Oliveira Duarte, "Sectored Receivers for Indoor Wireless Optical Communication Systems," 5th IEEE International Symposium on Personal Indoor and Mobile Radio Communications (PIMRC'94), The Netherlands, pp. 1090-1095, Sept. 1994. 